\title{
Intramasseteric schwannoma treated with facelift incision and retrograde facial nerve dissection
}

\author{
Jae Ha Hwang, \\ Dong Gyu Lee, \\ Ho Seup Sim, \\ Kwang Seog Kim, \\ Sam Yong Lee
}

Department of Plastic and

Reconstructive Surgery, Chonnam

National University Medical School,

Gwangju, Korea

\begin{abstract}
Schwannoma is a slow-growing, well-demarcated, benign soft tissue tumor of the peripheral nerve sheath. It commonly develops in the head and neck region, usually in the parapharyngeal space. In this case, a 42-year-old woman visited the outpatient department to manage a painless mass on her left cheek. She had no history of concern and no neurological symptoms were observed. In the enhanced computed tomography scan, a $2.8 \times 2.8 \times 1.8 \mathrm{~cm}$, heterogeneously enhanced tumor was detected in the left masseter muscle. A tumor resection under general anesthesia was planned. For the resection, a facelift incision was chosen; branches of the facial nerve were identified and retrogradely dissected. A well-marginated, yellowish, solid mass was found in the left masseter muscle. The mass was excised and given a histopathological diagnosis of schwannoma. A definite diagnosis of schwannoma, originating in the masseter muscle, is difficult to arrive at with radiographic findings alone; it is often misdiagnosed as intramuscular hemangioma. Histopathological examinations, including fine-needle aspiration or histological biopsy after surgery, are necessary. Using a facelift incision with retrograde facial nerve dissection, tumor resection in an intramasseteric lesion can be performed efficiently, without nerve damage, or leaving conspicuous scars on the face.
\end{abstract}

Keywords: Masseter muscle / Neurilemmoma / Rhytidoplasty

\section{INTRODUCTION}

Schwannoma, also known as neurinoma, neuroma, or neurilemmoma, is a benign soft tissue tumor originating from Schwann cells, which are the glial cells of the peripheral nervous system. The head and neck region is the most frequent site of schwannoma [1]. Extracranial non-vestibular schwannomas usually involve the VII, VIII and XII cranial nerves in the head and neck area. They develop mostly in the parapharyngeal space $[2,3]$. Only a few cases of intramasseteric schwannoma were described and they are often misdiagnosed as intramuscular hemangiomas due to their similar appearance on radio-

\section{Correspondence: Jae Ha Hwang}

Department of Plastic and Reconstructive Surgery, Chonnam National University

Medical School, 42 Jebong-ro, Dong-gu, Gwangju 61469, Korea

E-mail: psjhhwang@daum.net

Received October 4, 2019 / Revised October 8, 2019 / Accepted October 20, 2019 logical imaging $[4,5]$. When accessing the intramasseteric space, avoiding obvious scars on the face and keeping the branches and trunk of the facial nerve intact are crucial. This article describes a rare case of intramasseteric schwannoma that was treated with a facelift incision and retrograde facial nerve dissection.

\section{CASE REPORT}

A 42-year-old woman visited the outpatient department to manage a slowly growing, painless mass on her left cheek. It had been over 9 years since she first detected the lump on her preauricular area; its size had gradually increased. She had no noteworthy medical history, and no history of trauma or surgery. Upon physical examination, the mass was measured to be $3 \times 3 \mathrm{~cm}$, hardly movable, and non-tender. No cutaneous ab- 
normalities were observed above the tumor and regional lymphadenopathy was absent. Neurological symptoms, including sensory deficit, facial palsy, trismus, or impaired mastication, were not observed.

In the enhanced computed tomography (CT) scan, a $2.8 \times$ $2.8 \times 1.8 \mathrm{~cm}$ sized tumor was detected in the left masseteric muscle. It was hypodense compared to muscle and was heterogeneously enhanced. The clinical diagnosis made at this point was intramuscular hemangioma (Fig. 1).

It was decided that the tumor would be removed under general anesthesia. A facelift incision with pretragal and occipital incisions was chosen over a modified Blair incision to avoid a conspicuous scar. A skin and subcutaneous flap was raised through the facelift incision until the anterior border of the left parotid gland. The parotid duct and buccal and zygomatic branches of the facial nerve were identified. The gland was elevated from the masseter muscle and branches of the facial nerve were retrogradely dissected and preserved. The tumor was palpated above the masseter muscle and the muscle fibers were dissected. A $2.5 \times 2.2 \mathrm{~cm}$, yellowish, solid mass was found. It was well separated from the surrounding muscle fibers and was continuous with a small nerve branch. The mass was excised, along with a short segment of the nerve branch (Fig. 2).

The pathological examination showed characteristic Antoni A and Antoni B areas. Immunohistochemical staining was highly positive for $\mathrm{S} 100$ protein. Thus, the diagnosis was revised to schwannoma (Fig. 3).

After surgery, any sensory deficits or motor dysfunctions, including facial nerve palsy or impaired mastication, were not

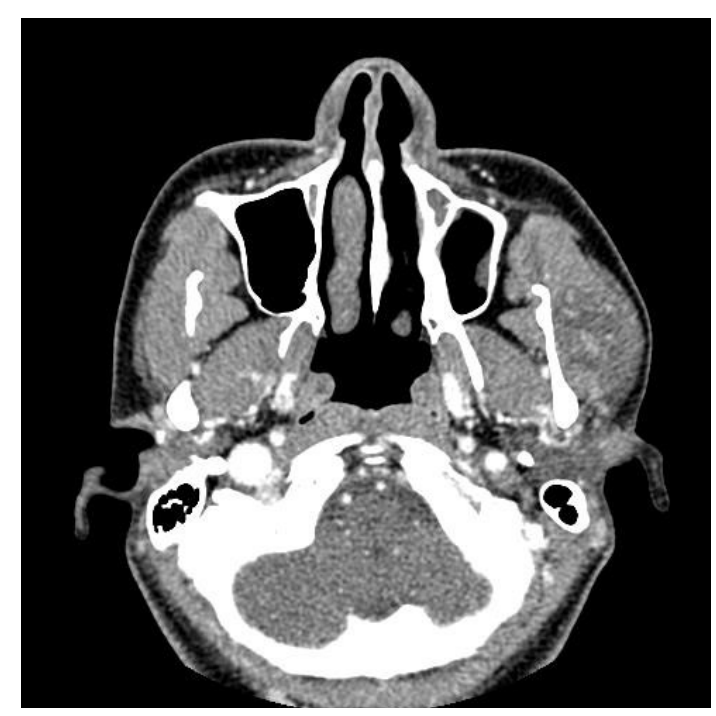

Fig. 1. In the enhanced computed tomography image, a $2.8 \times 2.8 \times 1.8$ $\mathrm{cm}$ heterogeneously enhancing mass in the left masseter muscle can be observed. observed. Clinical photography after 6 months shows a wellhidden scar with no tragal distortion (Fig. 4).

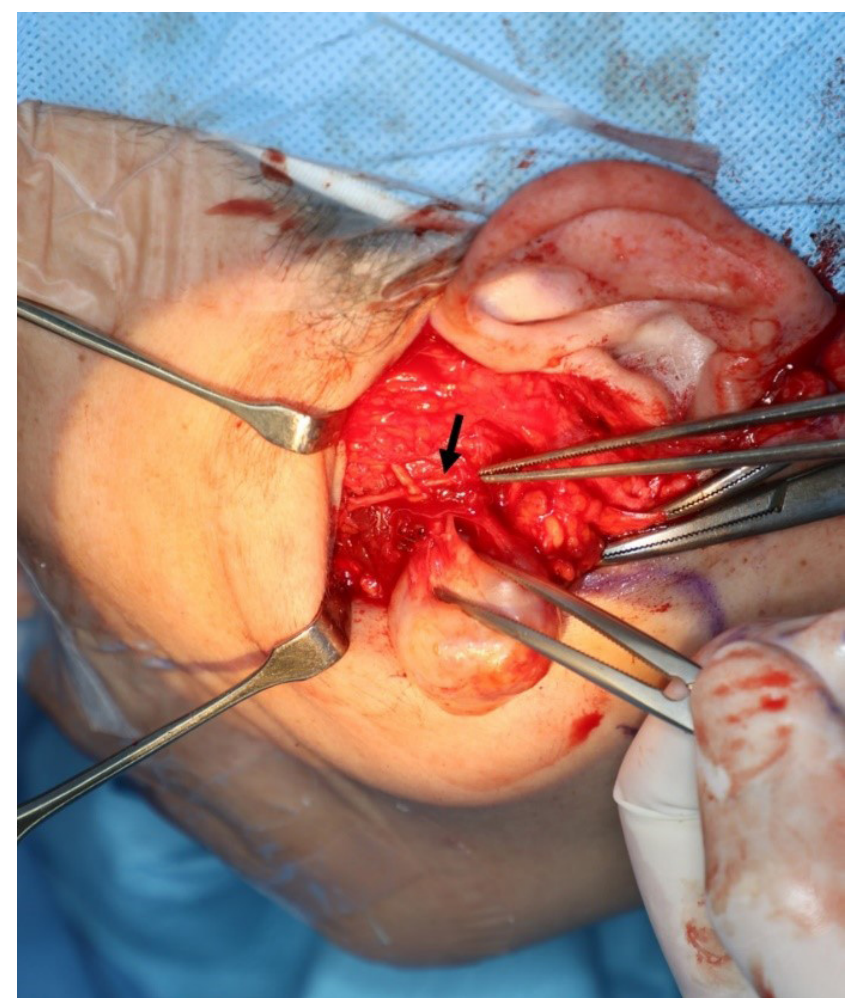

Fig. 2. In the operative field, a well-separated mass was found in the masseter muscle fibers. It was continuous with a small branch of nerves. The zygomatic branch of the facial nerve has been dissected and preserved (black arrow).

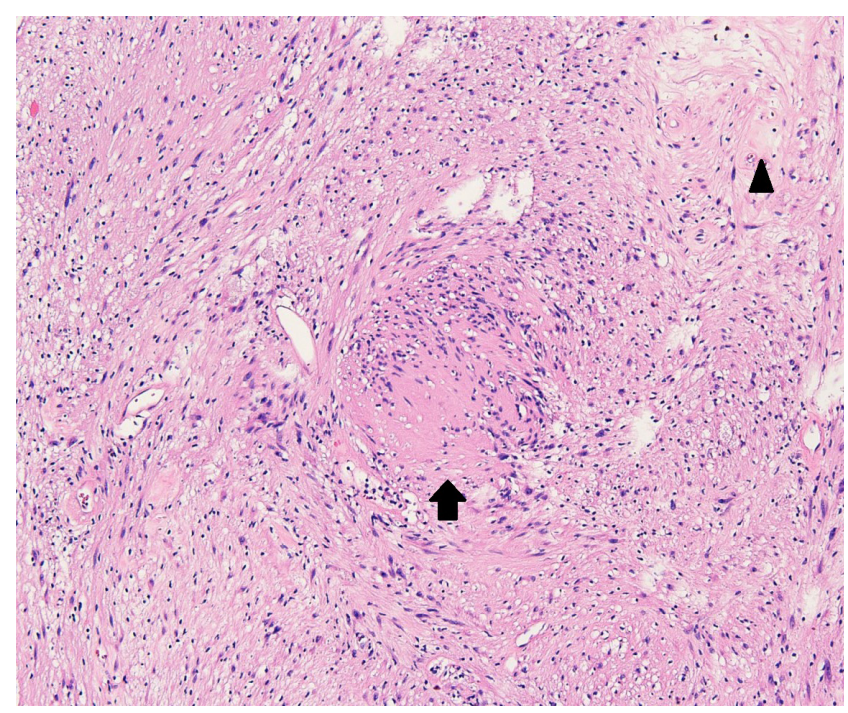

Fig. 3. Photomicrograph $(\mathrm{H} \& \mathrm{E}, \times 100)$ reveals that the tumor consists of focal cellular (Antoni A, black arrow) areas and hypocellular (Antoni B, black arrowhead) areas with vacuolar degeneration. 


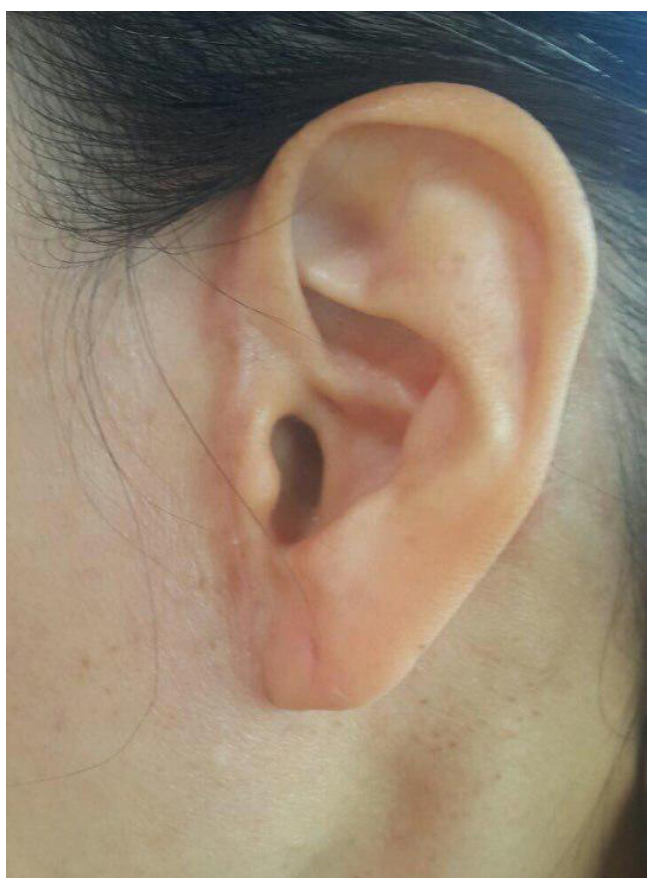

Fig. 4. A clinical photograph taken 6 months after excision. The facelift incision, with pretragal and occipital incision lines, was strategically placed and is well camouflaged without any tragal distortion.

\section{DISCUSSION}

Schwannoma is a benign soft tissue tumor that develops from Schwann cells, the glial cells of the peripheral nervous system. Schwannoma can be derived from any myelinated peripheral nerve (motor, sensory, or autonomic nerve) with the exception of the olfactory and optic nerves. The head and neck region is the most frequent site of schwannoma (25\%-45\%) [1]. Major studies focused mostly on intracranial acoustic schwannomas; however, a large proportion of schwannomas are extracranial non-vestibular and develop in the head and neck area. Extracranial non-vestibular schwannomas usually involve the VII, VIII, and XII cranial nerves and commonly reside in the parapharyngeal space $[2,3]$. Patients with schwannomas are usually within the ages of 20 to 50 years old; no sexual predominance is observed [3].

Both developmental and neoplastic mass lesions of the masseteric space are rare. Among developmental lesions, vascular malformations are common and several cases of intramasseteric hemangiomas have been described. For this diagnosis, a wide surgical excision is recommended because of high local recurrence rate. In some cases, preoperative embolization could be needed. Conversely, schwannoma is the most frequent neoplastic lesion in this region [6]. Typically, schwannomas are revealed as low-attenuated lesions in CT. In magnetic resonance imaging, they show a low or iso signal intensity, compared with muscle tissues, in T1-weighted images and a high signal intensity in T2-weighted images. These image findings are similar to those of hemangiomas. Therefore, by radiographic findings alone, it is difficult to diagnose schwannomas [4]. Fine-needle aspiration (FNA) may be helpful in preoperative diagnosis, however, its diagnostic accuracy varies strongly depending on the quality of the specimen and the experience of the cytopathologist [1]. In two cases of intramasseteric schwannomas misdiagnosed as hemangiomas, both of them performed FNA but neither of them drew a definite diagnosis [4,5]. Consequently, intramasseteric schwannomas are usually diagnosed by histological examination after surgical resection [4].

There are several surgical approaches to the intramasseteric region: through direct skin incision, intraoral approach, and skin flap approach, including the facelift or modified Blair incision methods. There are two main objectives in maneuvering these procedures. First, the goal is to avoid nerve damage and keep the facial nerve intact, considering the adjacency of the lesions to the parotid gland, which contains the trunk and branches of the facial nerve. Second, aesthetically, it is important to hide conspicuous scars. The direct skin incision method is technically simple, but it is likely to injure the branches of the facial nerve and leave an obvious scar on the face. The intraoral approach creates no visible scar; however, due to its confined operative cavity, the surgical technique is challenging and additional surgical instruments, including an endoscope, are often needed. Also, the ability to do this procedure often depends on the exact location of the intramasseteric lesion [6]. The skin flap approach, usually with facial nerve dissection, could keep the branches and trunk of the facial nerve intact. Using the facelift incision, noticeable scars on the face can be avoided. In this case, we considered that the intraoral approach would be difficult as the mass was large $(2.8 \times 2.8 \times 1.8 \mathrm{~cm})$ and positioned in the superoposterior aspect of the left masseter muscle.

The masseter muscle leaves the infratemporal fossa by passing through the mandibular notch. It is accompanied by a vascular pedicle from the inferior alveolar artery which has an average diameter of $2 \mathrm{~mm}$ [7]. According to its anatomy, the schwanno$\mathrm{ma}$, in this case, must have been derived from a branch of the masseteric nerve. Schwannomas are usually treated with complete surgical excision, aiming to preserve the affected nerve. Most clinicians have recommended a careful intracapsular excision of the tumor to minimize nerve injury; this involves microscopic enucleation inside the epineurium without disrupting neural continuity; although, partial resection of the tumor may be an option if significant morbidity from neural deficit is anticipated when the tumor is completely removed [1]. In this 
case, the nerve that was continuous with the tumor was likely the terminal branch of the masseter nerve; it was insignificantly small and considered to be inconsequential to the motor function of the masseter muscle. Instead of a tedious microscopic procedure, the tumor was excised along with a short segment of the nerve. The patient exhibited no functional abnormalities in mastication postoperatively. The en bloc nerve and tumor resection with tumor enucleation showed no difference in the recurrence rate; however, incomplete excision may lead to local recurrence [2,3]. In cases involving major nerves, microscopic enucleation to preserve neural integrity or immediate reconstruction of the nerve, if nerve fibers were compromised, should be undertaken.

Anterograde or retrograde facial nerve dissection can be performed. There is no difference in the rate of facial nerve recovery or the incidence rate of complications (e.g., temporal facial nerve palsy, Frey syndrome, hematoma, sialocele, wound infection, and postoperative hypertrophic scar) between the two procedures. However, retrograde facial nerve dissection has theological advantages. By selectively exposing the branches of the facial nerve, it could reduce the risk of nerve injury. Further, wide operative field make procedure easier with more meticulous hemostasis $[8,9]$. The merits of this procedure include a lessened operative time, decreased blood loss, and lesser removal of normal parotid tissue [10].

In this article, we presented a case of intramasseteric schwannoma. A definite diagnosis of schwannoma situated in the masseteric space is difficult with radiographic findings alone; histopathological examinations, including FNA or a histological biopsy after surgery, are necessary. Using a facelift incision with retrograde facial nerve dissection, the resection of the tumor in an intramasseteric lesion can be performed efficiently, without nerve damage, or leaving conspicuous scars on the face.

\section{NOTES}

\section{Conflict of interest}

No potential conflict of interest relevant to this article was reported.

\section{Ethical approval}

The study was approved by the Institutional Review Board of Chonnam National University Hospital (IRB No. CNUH-EXP2019-267) and performed in accordance with the principles of the Declaration of Helsinki. Written informed consent was obtained.

\section{Patient consent}

The patient provided written informed consent for the publication and the use of her images.

\section{ORCID}

Jae Ha Hwang https://orcid.org/0000-0001-6992-8067

Dong Gyu Lee https://orcid.org/0000-0003-3701-0395

Ho Seup Sim https://orcid.org/0000-0003-2911-0126

Kwang Seog Kim https://orcid.org/0000-0002-6766-4640

Sam Yong Lee https://orcid.org/0000-0002-3185-2519

\section{REFERENCES}

1. Kang GC, Soo KC, Lim DT. Extracranial non-vestibular head and neck schwannomas: a ten-year experience. Ann Acad Med Singapore 2007;36:233-8.

2. de Araujo CE, Ramos DM, Moyses RA, Durazzo MD, Cernea CR, Ferraz AR. Neck nerve trunks schwannomas: clinical features and postoperative neurologic outcome. Laryngoscope 2008;118:1579-82.

3. Malone JP, Lee WJ, Levin RJ. Clinical characteristics and treatment outcome for nonvestibular schwannomas of the head and neck. Am J Otolaryngol 2005;26:108-12.

4. He Y, Fu HH, He J, Zhu HG, Zhang ZY. Schwannoma arising from intramasseteric region. J Craniofac Surg 2010;21:19982001.

5. Nakamura A, Iguchi H, Kusuki M, Zushi T, Yamane H, Wakasa K. Intramasseteric schwannoma in a child. Auris Nasus Larynx 2006;33:347-50.

6. Aizawa T, Kubo S, Azuma R, Kiyosawa T. Approaches for excision of intramasseteric nodules. J Craniofac Surg 2019;30:e924.

7. Cotrufo S, Hart A, Payne AP, Sjogren A, Lorenzo A, Morley S. Topographic anatomy of the nerve to masseter: an anatomical and clinical study. J Plast Reconstr Aesthet Surg 2011;64:14249.

8. Anjum K, Revington PJ, Irvine GH. Superficial parotidectomy: antegrade compared with modified retrograde dissections of the facial nerve. Br J Oral Maxillofac Surg 2008;46:433-4.

9. O'Regan B, Bharadwaj G. Comparison of facial nerve injury and recovery rates after antegrade and retrograde nerve dissection in parotid surgery for benign disease: prospective study over 4 years. Br J Oral Maxillofac Surg 2011;49:286-91.

10. Bhattacharyya N, Richardson ME, Gugino LD. An objective assessment of the advantages of retrograde parotidectomy. Otolaryngol Head Neck Surg 2004;131:392-6. 